\title{
Tai-Chi and Baduanjin during treatment and rehabilitation of older adults with COVID-19
}

To the Editor,

In the COVID-19 outbreak, older adults have a higher infection rate because of their weak immune system. Tai-Chi and Baduanjin are common Qigong exercises that combined deep breathing and relaxation with flowing movements to improve respiratory muscles, whole-body movement, and mental rehabilitation.

Tai-Chi is a low-to-moderate intensity mindbody exercise for the treatment and prevention of infectious diseases. During the rehabilitation stage, Tai-Chi can minimise negative COVID-19 symptoms, including fever, asthenia, and cough, as well as shorten the hospitalisation time. ${ }^{1}$ Baduanjin combined with Chinese medicine increases the treatment and rehabilitation rates to higher than $87.5 \% .^{2}$ Practising Tai-Chi and Baduanjin can relax and change respiratory movement, rhythm, and pattern, and can restore the Qi (energy) in the body and move it to different internal organs such as the lung and spleen through meridians for better functions to enhance the immune system called 'defensive Wei Qi'. Therefore, practising Tai-Chi and Baduanjin can prevent and combat the virus invasion and its immunosuppression and cytokine storm syndromes.

The levels of IgA and IgG increase and the onset time of an upper respiratory tract infection shortened after 6 months training of Tai-Chi. ${ }^{3}$ Longer Tai-Chi exercise time significantly increased the levels of $\operatorname{Ig} \mathrm{A}, \operatorname{Ig} \mathrm{G}$, and IgM in the blood that enhance the immune system. ${ }^{4}$ The cell-mediated immunity was stronger after 25 weeks of practising Tai-Chi, ${ }^{5}$ and the response of antibody increased. ${ }^{6}$

Baduanjin is easier to learn for older people. It was reported that in older people the percentage of CD4, CD4/CD8, and NK cells increased and the immune system strengthened after 24 weeks of practising Baduanjin. ${ }^{7}$ The risk of respiratory tract infection was lowered by systematic exercise. ${ }^{8}$ Lung function such as forced expiratory volume in $1 \mathrm{~s}$, forced vital capacity rate of $1 \mathrm{~s}$, and arterial blood gas analysis improved for bronchitis patients after practising Baduanjin. ${ }^{9}$ Tai-Chi and Baduanjin are effective for older people during the treatment and rehabilitation stages of COVID-19 to promote recovery of the immune system.

\section{Siukan Law ${ }^{1}$, Albert Wingnang Leung ${ }^{2}$, Chuanshan $\mathrm{Xu}^{3}$}

1 Department of Science, School of Science and Technology, The Open University of Hong Kong, Ho Man Tin, Kowloon, Hong Kong

2 School of Graduate Studies, Lingnan University, Hong Kong

3 Key Laboratory of Molecular Target and Clinical

Pharmacology, State Key Laboratory of Respiratory Disease, School of Pharmaceutical Sciences \& Fifth Affiliated Hospital, Guangzhou Medical University, Guangzhou, China

Correspondence to: Dr Siukan Law, Department of Science, School of Science and Technology, The Open University of Hong Kong, Ho Man Tin, Kowloon, Hong Kong.

Email:siukanlaw@hotmail.com

\section{REFERENCES}

1. Shi Y, Wen D, Wang H, et al. Tai Chi for coronavirus disease 2019 in recovery period: a protocol for systematic review and metaanalysis. Medicine (Baltimore) 2020;99:e21459. Crossref

2. Han $\mathrm{YZ}, \mathrm{Fu}$ MW, Chen ZJ, et al. The effects of health Qigong Baduanjin on the prevention, treatment, and recovery of COVID-19. J Integr Nurs 2020;2:33-9. Crossref

3. Tie Y. Research on the impact of the Taichi Chuan or the upper respiratory tract infection for female students. Health Med Res Pract 2008;5:69-70

4. Niu A. Effect of "Tai Chi" exercise on antioxidant enzymes activities and immunity function in middle-aged participants. Afr J Tradit Complement Altern Med 2016;13:87-90.

5. Irwin MR, Olmstead R, Oxman MN. Augmenting immune responses to varicella zoster virus in older adults: a randomized, controlled trial of Tai Chi. J Am Geriatr Soc 2007;55:511-7. Crossref

6. Feng F, Tuchman S, Denninger JW, Fricchione GL, Yeung A. Qigong for the prevention, treatment, and rehabilitation of COVID-19 infection in older adults. Am J Geriatr Psychiatry 2020;28:812-9. Crossref

7. Yeh SH, Chuang H, Lin LW, Hsiao CY, Eng HL. Regular tai chi chuan exercise enhances functional mobility and CD4CD25 regulatory T cells. Br J Sports Med 2006;40:239-43. Crossref

8. Nieman DC. Exercise, upper respiratory tract infection, and the immune system. Med Sci Sports Exerc 1994;26:128-39. Crossref

9. Chen Y. Effect of BaDuanJin on respiratory symptoms in chronic bronchitis. Med Front 2016;6:361. 